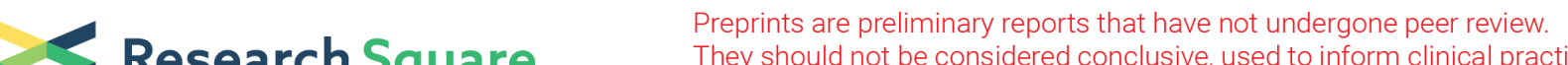 $\begin{array}{ll}\text { Research Square } & \text { They should not be considered conclusive, used to inform clinical practice, } \\ \text { or referenced by the media as validated information. }\end{array}$
}

\section{Diagnostic value of macrophage inhibitory cytokine 1 as a novel prognostic biomarkers for early gastric cancer screening}

\section{Xin Ge}

the first affiliated hospital of USTC

Xiaolei Zhang

the first affiliated hospital of USTC

Yanling Ma

Anhui office for cancer control and research

Shaohua Chen

the first affiliated hospital of USTC

Zhaowu Chen

the first affiliated hospital of USTC

Ming Li ( $\nabla$ nothing614@163.com )

University of Science and Technology of China

Research article

Keywords: Gastric cancer, MIC-1, biomarkers

Posted Date: March 26th, 2020

DOI: https://doi.org/10.21203/rs.3.rs-17962/v1

License: () (1) This work is licensed under a Creative Commons Attribution 4.0 International License.

Read Full License

Version of Record: A version of this preprint was published at Journal of Clinical Laboratory Analysis on September 12th, 2020. See the published version at https://doi.org/10.1002/jcla.23568. 


\section{Abstract}

BACKGROUND Early diagnosis is very important to improve the survival rate of patients with gastric cancer, especially in asymptomatic participants. However, low sensitivity of common biomarkers has caused difficulties in early screening of gastric cancer. In this study, we explored whether MIC-1 can improve the detection rate of early gastric cancer.

METHODS We screened 8,257 participants based on risk factors such as age, gender, and family history for physical examination including gastroscopy. Participant blood samples were taken for measure MIC1, CA-199, CA72-4 and PG1/PG2 levels. The diagnostic performance of MIC-1 was assessed and compared with CA-199, CA72-4 and PG1/PG2, and its role in early gastric cancer diagnosis and the assessment of the risk of precancerous lesions have also been studied.

RESULTS Based on endoscopic and histopathological findings, 55 participants had gastric cancer, 566 participants had low-grade neoplasia, 2605 participants had chronic gastritis. MIC-1 levels were significantly elevated in gastric cancer serum samples as compared to controls $(p<0.001)$. The sensitivity of serum MIC-1 for gastric cancer diagnosis was much higher than that of CA-199 (49.1\% vs. $20.0 \%)$ with similar specificities. Moreover, receiver operating characteristic (ROC) curve analysis also showed that serum MIC-1 had a better performance compared with CA-199, CA72-4 and PG1/PG2 in distinguishing early-stage gastric cancer (AUC: $72.9 \%$ vs. $69.5 \%, 67.5 \%, 44.0 \%$ respectively).

CONCLUSIONS Serum MIC-1 is significantly elevated in most patients with early gastric cancer. MIC-1 can serve as a novel diagnostic marker of early gastric cancer and value the risk of gastric cancer.

\section{Background}

Although the incidence of gastric cancer (GC) has declined in some developed countries, it continues to be an important healthcare problem from a global perspective [1]. Due to poor standards of hygiene, more than $70 \%$ of gastric cancers occur in developing countries, and gastric cancer is the third leading cause of cancer-related death in China [2-3]. Despite advances in therapeutic approaches for gastric cancer, clinical prognosis with a reported five-year survival rate was less than $30 \%$. Therefore, the most critical means to reduce the incidence of gastric cancer is the improvement of early diagnosis rate.

Histological study of gastric mucosa is the most accurate method for diagnosing gastric cancer, but the popularity of endoscopic is very low due to the invasive nature, especially in asymptomatic patients. So, serum tumor biomarkers play an important role in gastric cancer screening in the past decades. As the lack of unique biomarker, combined detection of multiple serological biomarkers was used for predicting risk of gastric cancer [4]. Carcinoembryonic antigen (CEA), pepsinogen (PG), carbohydrate antigen 724 (CA 724) and carbohydrate antigen 199 (CA 199) as GC biomarkers have been studied widely[5-7]. However, there are many problems with the specificity and sensitivity of joint diagnosis, so it is particularly important to find a specific biomarker that can improve the diagnosis of early gastric cancer [8]. 
Macrophage inhibitory cytokine-1(MIC-1), as a member of the transforming growth factor beta superfamily, is detected in the inflammatory response and tissue repair after acute injury initially [9]. Serum MIC-1 is elevated in many cancer patients and is associated with tumor pathogenesis, progression and invasion, such as lung cancer, colorectal cancer, pancreatic cancer, etc [10-12]. There have been related studies between MIC-1 and gastric cancer, but they focus on related pathogenic mechanisms, while the role of MIC-1 in early gastric cancer screen is still unknown [11-13].

The purpose of the current study was to determine whether MIC-1 can improve the detection rate of gastric cancer in asymptomatic participants compared with other traditional indicators.

\section{Methods}

\section{Study Population}

The residents of Hefei City, Anhui Province participated in a medical examination program for gastric cancer screening from the west branch of the First Affiliated Hospital of the University of Science and Technology of China from 2014 to 2017. Participants were asymptomatic adults aged over 40 years old and had no prior history of any cancer. For each individual visiting for medical examination, relevant clinical notes, previous operations, family history of all types of cancer in first-degree relatives, and other risk factors for gastric cancer including cigarette smoking were documented. All participants were enrolled into the study only after written informed consent.

\section{Screening Endoscopic}

Endoscopic was offered to all individuals who joined gastric cancer screening. The individuals were fasted for at least six hours and one of the lidocaine mucilage took 10 minutes before the endoscopic examination. All participants' blood pressure was guaranteed blow 140/90 mmHg. After endoscope was inserted into the esophagus of examiner, the order of esophageal- cardiac-gastric-pyloric-duodenum examination was performed. Mucosal color, smoothness, mucus, peristalsis and lumen shape were observed to determine the presence of lesions. If the lesion was found, it was necessary to record the shape, extent, location, and cytology organization. The tissue was further stained with hematoxylin and eosin in the pathology department to determine the nature of the lesion and classification.

\section{MIC-1 ELISA}

The serum of all participants was collected. The patients diagnosed as gastric cancer and high-grade neoplasia were defined as the group of gastric cancer and 52 healthy subjects were randomly selected as the control group. Serum MIC-1 was detected by a sensitive ELISA, which was produced by CICAMS (cancer institute and hospital, Chinese Academy of Medical Sciences) as detailed previously [15]. All samples were analyzed using ELISA assays on the same day. All serum samples were duplicately assayed. 
The serum levels of CA-199, CA72-4, PG1 and PG2 were tested by electrochemiluminescence immunoassay (ECLIA) kits using Elecsys 601 (Roche, America). All serum samples were duplicately assayed.

\section{Statistics}

The chi-square test was used to investigate associations between MIC-1 level and clinical characteristics of gastric cancer patients. ROC curve was used to identify the diagnostic information. Multivariable logistic regression model was conducted to corporate diagnostic performance of biomarkers. The clinical cut-off value for MIC-1 was assigned as the mean value plus two point five standard deviations of healthy individuals, and the clinical cut-off values for CA-199, CA72-4, PG1 and PG2 were based on the manufacturer's protocols. The statistical activity was operated with SPSS 16.0. $P$ value of $<0.05$ for a two-sided test was considered to be statistically significant.

\section{Results}

\section{Clinic pathological characteristics of the study population}

Among the 8257 participants (5340 males; 4717 females), 55 had gastric cancer including 27 with highgrade neoplasia, 18 with early gastric cancer and 10 with advanced gastric cancer. The baseline characteristics of the subjects who underwent an endoscopic examination are presented in Table1. The features indicative of gastric cancer are listed in Table2.

\section{Risk factors for gastric cancer development}

The risk factors for gastric cancer were shown in Table3. According to a univariate analysis, age, sex and family history were significant. Smoking history and BMI cannot be risk factors for gastric cancer.

\section{Early diagnostic value of MIC-1 for gastric cancer}

In this study, we classified high-grade neoplasia into the gastric cancer group, and most gastric cancers were in early stage, so we evaluated the value of serum MIC-1 as a marker for early diagnosis of gastric cancer compared with traditional biomarkers. We randomly selected 52 healthy subjects as negative controls from the population participating in the study. Interestingly, the serum level of CA-199 $(p=0.097)$, PG1/PG2 ( $p=0.303)$ did not differ significantly in gastric cancer patients compared to healthy controls but CA72-4 ( $p=0.022)$ and MIC-1 $(p<0.001)$ level was significantly increased in gastric cancer patients (Figure1).

Next, Receiver Operating Characteristic Curves (ROC) was used to evaluate the advantage of MIC-1 as serum diagnostic markers for early stage gastric cancer. The area under ROC curve (AUC) of CA-199, CA72-4 and PG1/PG2 were $0.695,0.675$ and 0.440 , respectively. Compared to traditional biomarkers, MIC1 (AUC: 0.729 ) had the better ability to distinguish gastric cancer cases from healthy subjects (Figure2). 
We further calculated the detection sensitivity of MIC-1 for gastric cancer at various specificity cut-offs and set $435.66 \mathrm{pg} / \mathrm{ml}$ as the cutoff value for warranting acceptable specificity. Table 4 showed the diagnostic value of MIC-1, CA-199, CA72-4, PG1/PG2 and combined biomarkers. The sensitivity of MIC-1 was $49.09 \%$ and significantly higher than that of CA-199 (20.00\%), CA72-4 (21.82\%). Although PG1/PG2 showed similar sensitivity to MIC-1, its specificity (63.46\%) was much lower than MIC-1(90.38\%). These data revealed that serum MIC-1 separate detection was superior to other biomarkers in gastric cancer detection, especially early-stage gastric cancer.

\section{Risk factors for gastric cancer according to MIC-1 level}

Analysis by chi-square test, no significant correlation was identified between MIC-1 level and other factors (Table5). Relevant data suggested MIC-1 can be an independent risk factor for early gastric cancer.

\section{Discussion}

Gastric cancer is one of the most malignant tumors, and its mortality and morbidity are increasing year by year in China. Despite the variation of practice pattern worldwide, surgical resection of early and midstage gastric cancer patients is still recognized as the most effective method. However, the lack of typical clinical symptoms and the intolerance of endoscopy leading delay in gastric cancer diagnosis. Most patients with clinical signs have been diagnosed as advanced gastric cancer and have lost optimal surgical timing. Therefore, early detection and diagnosis of gastric cancer are extremely important in improving the survival of the patients.

Tumor biomarkers are highly correlated with tumorigenesis, diagnosis, recurrence and prognosis. Finding a specific serum biomarker for tumor has always been the focus of research such as the specificity of alpha-fetoprotein for liver cancer diagnosis. There are many serum biomarkers of gastric cancer currently used internationally and the common ones are CEA, CA-199, CA72-4 and PG1/PG2. However, the sensitivity and specificity are not satisfactory, especially for early stage gastric cancer. The serum levels of CEA, CA19-9, and CA72-4 may be elevated in patients with gastric cancer at various stages [16]. The National Comprehensive Cancer Network guidelines do not recommend serum marker testing for preoperative evaluation and staging of gastric cancer. Therefore, an additional biomarker favoring early detection and diagnosis of gastric cancer is still urgently needed.

Macrophage inhibitory cytokine 1 (MIC-1/GDF15), a 25-kDa secreted growth factor of transforming growth factor- $\beta$ (TGF- $\beta$ ) super-family, was originally discovered in macrophage cells [17-18]. In previous studies, overexpression of MIC-1 in gastric cancer tissues was associated with tissue differentiation, and high levels of MIC-1 predicted high potential invasiveness [19]. Although previous studies have reported that the serum MIC-1 level in patients with gastric cancer was higher than healthy people, they have concluded that MIC-1 is associated with gastric cancer progression and prognosis [20-21]. The diagnostic value of MIC-1 in early gastric cancer has not been investigated comprehensively. 
The present study is the first to evaluate the clinical value of MIC-1 in early gastric cancer based on largescale investigation. We analyzed the expression of MIC-1 in gastric cancer serum, and found that MIC-1 showed a significant difference in levels between gastric cancer patients and healthy controls $(P<0.001)$, with no noticeable difference observed for CA-199, CA72-4 or PG1/PG2 $(P>0.10)$. Compared with the biomarkers of CA-199, CA72-4 and PG1/PG2, MIC-1 appears to hold the most individual promise in differentiating patients with gastric cancer from those without, giving sensitivities of $49.09 \%$, specificities of $90.38 \%$, and areas under the curve (AUCs) of 0.729 . Interestingly, there is no correlation between MIC-1 and high risk factors of gastric cancer, suggesting that high MIC-1 level can be used as an independent risk factor for early gastric cancer.

\section{Conclusions}

In summary, our research confirms that MIC-1 can be a new ideal tumor biomarker for early diagnosis of gastric cancer. Based on our present findings, future study is warranted for the development of relationship between MIC-1 and gastric cancer staging, treatment effect detection, recurrence and metastasis. In this manner, MIC-1 may be evaluated for its superiority to serum as a source for biomarkers in gastric cancer.

\section{Abbreviations}

GC

gastric cancer

CEA

Carcinoembryonic antigen

PG

pepsinogen

CA 724

carbohydrate antigen 724

CA 199

carbohydrate antigen 199

MIC-1

Macrophage inhibitory cytokine-1

\section{Declarations}

\section{Availability of data and materials}

All data and materials are not available in this study, and are available from the corresponding author on reasonable requests.

Acknowledgements 
Not applicable

\section{Funding}

This work was supported by The Fundamental Research Funds for the Central Universities (No. WK9110000025) to Ming Li.

\section{Authors' Contributions}

GX performed the study, analyzed the results, and drafted the manuscript. XL Zhang designed the study, and edited the manuscript. ZW Chen and SH Chen performed sample collection. LM contributed to strategic development decisions. All authors have read and approved this version of the article. Neither the entire paper nor any part of its content has been published or has been accepted elsewhere.

\section{Ethics declarations}

\section{Ethics approval and consent to participate}

This study was approved by the Medical Ethical Committee of the First Affiliated Hospital of USTC. All patients had signed informed consent.

\section{Consent for publication}

Not applicable.

\section{Competing interests}

Authors declare that they have no competing interests.

\section{References}

[1] Hudler P: Challenges of deciphering gastric cancer heterogeneity. World J Gastroenterol 21: 10510-10527, 2015.

[2] Jemal A, Bray F, Center MM, et al. Global cancer statistics. CA Cancer J Clin 2011; 61: 69-90

[3] Ferlay J, Shin H-R, Bray F, et al. Estimates of worldwide burden of cancer in 2008: GLOBOCAN 2008. Int J Cancer 127(12):2893-2917

[4] Rugge M, Capelle LG, Cappellesso R, Nitti D, Kuipers EJ.

Precancerous lesions in the stomach: from biology to clinical patient management. Best Pract Res Clin Gastroenterol 2013;27: 205-223

[5] Pectasides D, Mylonakis A, Kostopoulou M, et al. CEA, CA 19-9 and CA-50 in monitoring gastric carcinoma. Am J Clin Oncol 20: 348-353, 1997. 
[6] Lee HS, Jeon SW, Nomura S, et al. Screening Biomarker as an Alternative to Endoscopy for the Detection of Early Gastric Cancer: The Combination of Serum Trefoil Factor Family 3 and Pepsinogen. Gastroenterol Res Pract. 2018 May 9; 2018: 1024074.

[7] Shimada H, Noie T, Ohashi M, Oba K, Takahashi Y. Clinical significance of serum tumor markers for gastric cancer: a systematic review of literature by the Task Force of the Japanese Gastric Cancer Association. Gastric Cancer. 2014 Jan;17(1):26-33.

[8] Yang AP, Liu J, Lei HY, Zhang QW, Zhao L and Yang GH:CA72-4 combined with CEA, CA125 and CAl9-9 improves thesensitivity for the early diagnosis of gastric cancer. Clin ChimActa 437: 183-186, 2014.

[9] Bootcov MR, Bauskin AR, Valenzuela SM, et al. MIC-1, a novel macrophage inhibitory cytokine, is a divergent member of the TGF-beta superfamily. Proc Natl Acad Sci U S A. 1997; 94:11514-11519.

[10] Koopmann J, Buckhaults P, Brown DA, et al. Serum macrophage inhibitory cytokine 1 as a marker of pancreatic and other periampullary cancers. Clin Cancer Res. 2004; 10:2386-2392.

[11] Liu YN, Wang XB, Wang T, et al. Macrophage Inhibitory Cytokine-1_as a Novel Diagnostic and Prognostic Biomarker in Stage I and II Nonsmall Cell_Lung Cancer. Chin Med J (Engl). 2016 Sep 5;129(17):2026-32.

[12] Vocka M, Langer D, Fryba V, et al. Growth/differentiation factor 15 (GDF-15) as new potential serum marker in patients with metastatic_colorectal cancer. Cancer Biomark. 2018; 21(4): 869-874.

[13] Kim KK, Lee JJ, Yang Y, You KH, Lee JH. Macrophage Inhibitory Cytokine-1 activates AKT and ERK$1 / 2$ via the transactivation of ErbB2 in human breast and gastric cancer.

Carcinogenesis. 2008 Apr;29(4):704-12.

[14] Lee DH, Yang Y, Lee SJ, et al. Macrophage Inhibitory Cytokine-1 induces the invasiveness of gastric cancer cells by up-regulating the urokinase-type plasminogen activator system. Cancer Res. 2003 Aug 1;63(15):4648-55.

[15] Wang XB, Jiang XR, Yu XY, et al. Macrophage inhibitory factor 1 acts as a potential biomarker in patients with esophageal squamous cell carcinoma and is a target for antibody-based therapy. Cancer Sci. 2014; 105:176-85.

[16] Kim DH, Oh SJ, Oh CA, et al. The relationships between perioperative CEA, CA 19-9, and CA 72-4 and recurrence in gastric cancer patients after curative radical gastrectomy. J Surg Oncol. 2011;104:58591.

[17] Bootcov MR, Bauskin AR, Valenzuela SM, et al. MIC-1, a novel macrophage inhibitory cytokine, is a divergent member of the TGF-beta superfamily. Proc Natl Acad Sci U S A 1997, 94(21):11514-11519. 
[18] Paralkar VM, Vail AL, Grasser WA, et al. Cloning and characterization of a novel member of the transforming growth factor-beta/bone morphogenetic protein family. J Biol Chem 1998, 273(22):1376013767.

[19] Lee DH, Yang Y, Lee SJ, et al. Macrophage inhibitory cytokine-1 induces the invasiveness of gastric cancer cells by up-regulating the urokinase-type plasminogen activator system [J]. Cancer Res, 2003, 63:4648.

[20] Badreddine R, Wang KK. Biomarkers in Gastrointestinal Cancer [J]. Am J Gastroenterol, 2008, 103(8):2106.

[21] Baek KE, Yoon SR, Kim JT, et al. Upregulation and secretion of macrophage inhibitory cytokine-1 (MIC-1) in gastric cancers [J]. Clin Chim Acta, 2009, 401:128.

\section{Tables}

Table I Clinical characteristics of study population

\begin{tabular}{lcc}
\hline Characteristic & $\mathrm{n}$ & $(\%)$ \\
\hline Age & & \\
$\quad \leq$ Median age & 4348 & 52.66 \\
$\quad>$ Median age & 3909 & 47.34 \\
Sex & & \\
$\quad$ Female & 4717 & 57.13 \\
$\quad$ male & 3540 & 42.87 \\
Smoking status & & \\
$\quad$ Never smokers & 5208 & 63.07 \\
$\quad$ Current smokers & 1990 & 24.10 \\
$\quad$ Former smokers & 540 & 6.54 \\
$\quad$ Passive smoking & 519 & 6.29 \\
BMI, kg/m & & \\
$\quad<25$ & 5614 & 67.99 \\
$\quad \geq 25$ & 2643 & 32.01 \\
Family history (lung cancer) & & \\
$\quad$ Yes & 177 & 2.14 \\
$\quad$ No & 8080 & 97.86 \\
Chronic gastritis & 2605 & 31.55 \\
Low-grade neoplasia & 566 & 6.85 \\
Gastric cancer & 55 & 0.67 \\
\hline
\end{tabular}

Table II Clinical characteristics of gastric cancer 


\begin{tabular}{|c|c|c|}
\hline Gastric cancer $(\mathrm{n}=55)$ & $\mathrm{n}$ & $(\%)$ \\
\hline \multicolumn{3}{|l|}{$\overline{\text { age }}$} \\
\hline$\leq$ Median age & 15 & 27.27 \\
\hline$>$ Median age & 40 & 72.73 \\
\hline \multicolumn{3}{|l|}{ Sex } \\
\hline Female & 15 & 27.27 \\
\hline male & 40 & 72.73 \\
\hline \multicolumn{3}{|l|}{ Smoking status } \\
\hline Never smokers & 34 & 61.82 \\
\hline Current smokers & 13 & 23.64 \\
\hline Former smokers & 4 & 7.27 \\
\hline Passive smoking & 4 & 7.27 \\
\hline \multicolumn{3}{|l|}{ BMI, $\mathrm{kg} / \mathrm{m}^{2}$} \\
\hline$<25$ & 32 & 58.18 \\
\hline$\geq 25$ & 23 & 41.82 \\
\hline \multicolumn{3}{|l|}{ Family history (lung cancer) } \\
\hline Yes & 13 & 23.64 \\
\hline No & 42 & 76.36 \\
\hline \multicolumn{3}{|l|}{ Stage } \\
\hline High-grade neoplasia & 27 & 49.09 \\
\hline Early Gastric cancer & 18 & 32.73 \\
\hline Advanced Gastric cancer & 10 & 18.18 \\
\hline
\end{tabular}

Tablel The risk factors for gastric cancer 


\begin{tabular}{|c|c|c|c|c|}
\hline \multirow[b]{2}{*}{ Variables } & \multicolumn{4}{|c|}{ Univariate } \\
\hline & $\begin{array}{c}\text { Control } \\
(\mathrm{n}, \%)\end{array}$ & $\begin{array}{c}\text { Cancer } \\
(\mathrm{n}, \%)\end{array}$ & $\chi^{2}$ & $P$-Value \\
\hline age & & & 14.313 & 0.000 \\
\hline$\leq$ Median age & 4333 & 15 & & \\
\hline$>$ Median age & 3869 & 40 & & \\
\hline Sex & & & 20.149 & 0.000 \\
\hline Female & 4702 & 15 & & \\
\hline male & 3500 & 40 & & \\
\hline Smoking status & & & 0.453 & 0.939 \\
\hline Never smokers & 5174 & 34 & & \\
\hline Current smokers & 1977 & 13 & & \\
\hline Former smokers & 536 & 4 & & \\
\hline Passive smoking & 515 & 4 & & \\
\hline BMI, $\mathrm{kg} / \mathrm{m}^{2}$ & & & 2.448 & 0.118 \\
\hline$<25$ & 5582 & 32 & & \\
\hline$\geq 25$ & 2620 & 23 & & \\
\hline Family history (lung cancer) & & & 111.833 & 0.000 \\
\hline Yes & 164 & 13 & & \\
\hline No & 8038 & 42 & & \\
\hline
\end{tabular}

Table[ Performance characteristics of tumor markers

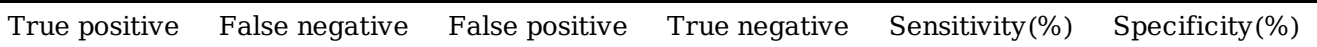

\begin{tabular}{|c|c|c|c|c|c|c|}
\hline CA199 & 11 & 44 & 1 & 51 & 20.00 & 98.08 \\
\hline CA724 & 12 & 43 & 3 & 49 & 21.82 & 94.23 \\
\hline 'G1/PG2 & 28 & 27 & 19 & 33 & 50.91 & 63.46 \\
\hline MIC1 & 27 & 28 & 5 & 47 & 49.09 & 90.38 \\
\hline 199+CA724 & 19 & 36 & 3 & 49 & 34.55 & 94.23 \\
\hline :A724+ PG1/PG2 & 32 & 23 & 21 & 31 & 58.18 & 59.62 \\
\hline PG2+MIC1 & 41 & 14 & 22 & 30 & 74.55 & 57.69 \\
\hline :4+ PG1/ PG2+MIC1 & 43 & 12 & 24 & 28 & 78.18 & 53.85 \\
\hline
\end{tabular}

Table $[$ Characteristics of the gastric cancer patients according to MIC1 status 


\begin{tabular}{|c|c|c|c|c|}
\hline \multirow[t]{2}{*}{ Variables } & \multicolumn{2}{|c|}{ MIC1 } & \multirow[t]{2}{*}{$x^{2}$} & \multirow[t]{2}{*}{$P$} \\
\hline & $(\leq 435.66)$ & $(>435.66)$ & & \\
\hline \multicolumn{5}{|l|}{ age } \\
\hline$\leq$ Median age & 12 & 6 & 2.658 & 0.103 \\
\hline >Median age & 16 & 21 & & \\
\hline \multicolumn{5}{|l|}{ Sex } \\
\hline Female & 6 & 9 & 0.982 & 0.322 \\
\hline male & 22 & 18 & & \\
\hline \multicolumn{5}{|l|}{ Smoking status } \\
\hline Never smokers & 18 & 16 & 2.082 & 0.586 \\
\hline Current smokers & 6 & 7 & & \\
\hline Former smokers & 1 & 3 & & \\
\hline Passive smoking & 3 & 1 & & \\
\hline $\mathrm{BMI}, \mathrm{kg} / \mathrm{m}^{2}$ & & & 0.025 & 0.874 \\
\hline$<25$ & 16 & 16 & & \\
\hline$\geq 25$ & 12 & 11 & & \\
\hline Family history (lung cancer) & & & 0.154 & 0.695 \\
\hline No & 22 & 20 & & \\
\hline Yes & 6 & 7 & & \\
\hline \multicolumn{5}{|l|}{ Stage } \\
\hline High-grade neoplasia & 15 & 12 & 0.538 & 0.764 \\
\hline Early Gastric cancer & 8 & 10 & & \\
\hline Advanced Gastric cancer & 5 & 5 & & \\
\hline \multicolumn{5}{|l|}{ CA199 } \\
\hline$\leq 27$ & 24 & 20 & 1.164 & 0.281 \\
\hline$>27$ & 4 & 7 & & \\
\hline CA724 & & & 1.897 & 0.168 \\
\hline$\leq 8.2$ & 24 & 19 & & \\
\hline$>8.2$ & 4 & 8 & & \\
\hline PG1/PG2 & & & 0.019 & 0.891 \\
\hline$\leq 3$ & 14 & 14 & & \\
\hline$>3$ & 14 & 13 & & \\
\hline
\end{tabular}

Figures 

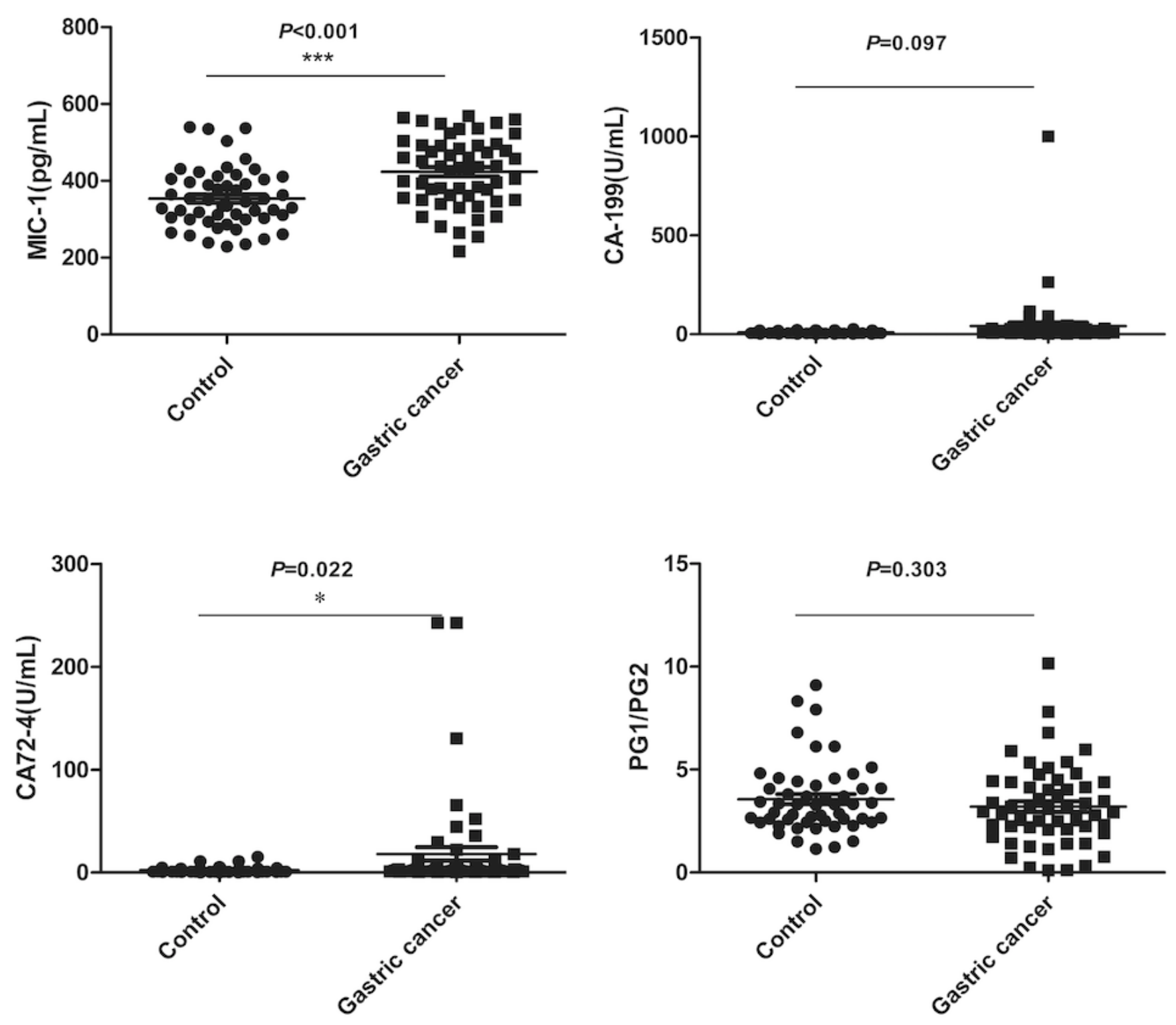

Figure 1

T-test of gastric cancer patients compared to healthy controls. 


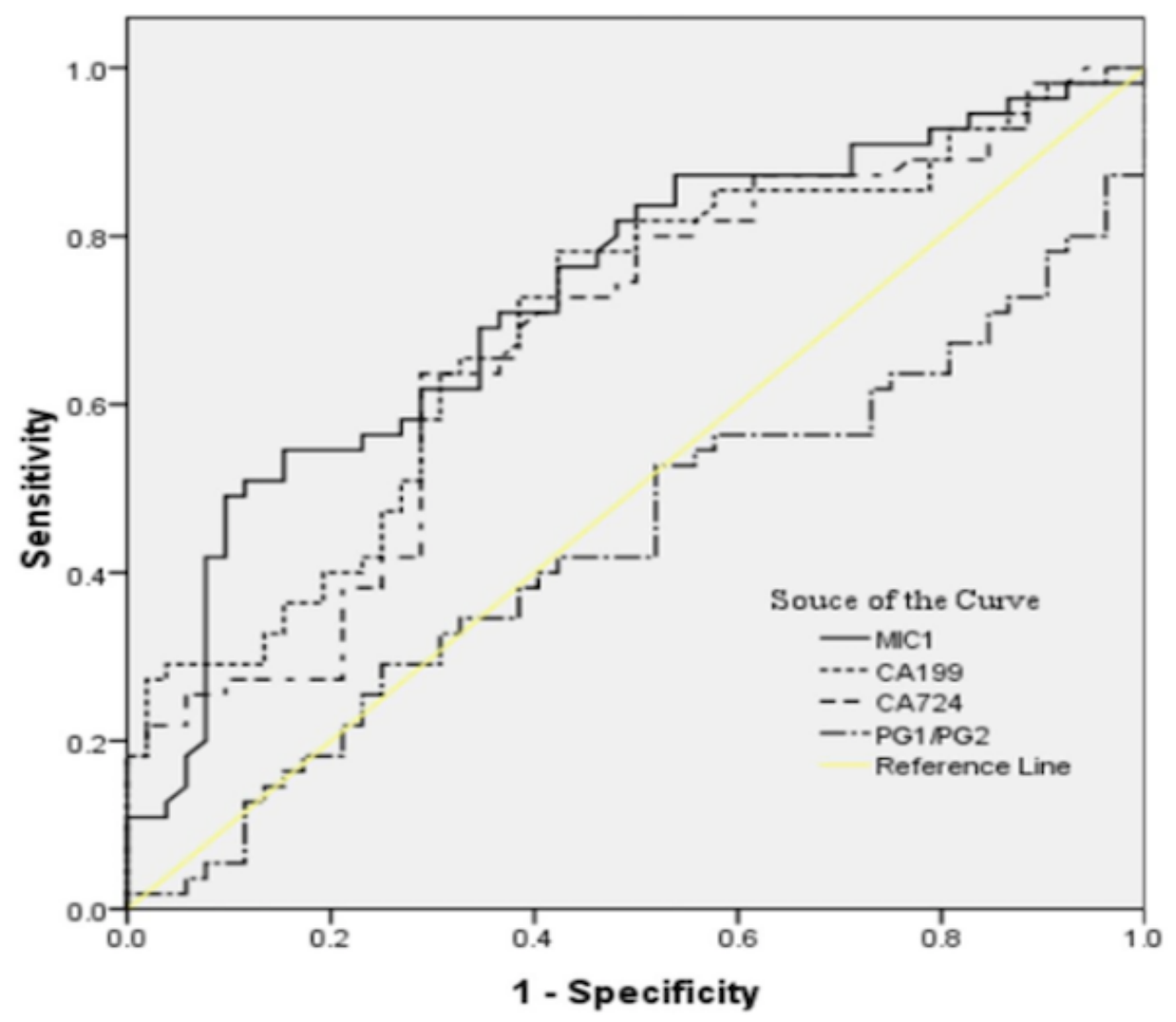

Area under receiver operating characteristic curve (AUROC)

\begin{tabular}{|c|c|c|c|c|c|}
\hline & \multirow{2}{*}{ AUC } & \multirow{2}{*}{ SE } & \multirow{2}{*}{ Pvalue } & \multicolumn{2}{|c|}{$95 \%$ CI } \\
\cline { 5 - 6 } & & & & lower & up \\
\hline MIC1 & 0.729 & 0.049 & $<0.001$ & 0.633 & 0.825 \\
\hline CA199 & 0.695 & 0.051 & 0.001 & 0.595 & 0.795 \\
\hline CA724 & 0.675 & 0.052 & 0.002 & 0.573 & 0.777 \\
\hline PG1/PG2 & 0.440 & 0.056 & 0.281 & 0.330 & 0.549 \\
\hline
\end{tabular}

Figure 2

The area under ROC curve (AUC) of CA-199, CA72-4 and PG1/PG2 